(2) Open Access Full Text Article

\title{
Spectral domain optical coherence tomographic findings at convalescent stage of acute zonal occult outer retinopathy
}

This article was published in the following Dove Press journal:

Clinical Ophthalmology

17 July 2009

Number of times this article has been viewed

\section{Kouichi Ohta \\ Atsuko Sato \\ Emi Fukui}

Department of Ophthalmology, Matsumoto Dental University, Shiojiri, Nagano, Japan
Correspondence: Kouichi Ohta Department of Ophthalmology, Matsumoto Dental University, 1780 Gobara, Hiooka, Shiojiri, 399-078I,

Nagano, Japan

Tel +81263512210

$\mathrm{Fax}+81263512210$

Email ohta@po.mdu.ac.jp
Purpose: To describe the morphology of the retina at the convalescent stage of acute zonal occult outer retinopathy (AZOOR) from images obtained by spectral domain optical coherence tomography (SD-OCT).

Methods: The visual fields, electroretinograms (ERGs), and OCT images were reviewed in two women aged 24 and 33 years. The patients were followed for one and four years, respectively.

Results: In both cases, the anterior and posterior segments were almost normal, although both patients had a sudden unilateral vision decrease and photopsia. Goldmann perimetry revealed enlarged blind spots and scotomas. The ERGs were reduced in both cases. SD-OCT showed that the junction of the inner and outer segment, the IS/OS line, of the photoreceptors was irregular or lost in the affected retinas. The retina in these areas was thinner due to a decrease in the thickness of both the outer nuclear layer (ONL) and inner nuclear layer (INL) in Case 2.

Conclusions: The decrease in retinal thickness at the convalescent stage of AZOOR is most likely due to a shortening of not only the photoreceptors and ONL but also to a thinning of the INL in a severe case.

Keywords: AZOOR, SD-OCT, IS/OS, ONL

\section{Introduction}

Acute zonal occult outer retinopathy (AZOOR) occurs in younger women, and is characterized by an acute decrease of retinal function of the outer layers of the retina, photopsia, minimal funduscopic changes, and electroretinographic (ERG) abnormalities. ${ }^{1}$ The changes can be unilateral or bilateral. ${ }^{1}$ Optical coherence tomography (OCT) of eyes with AZOOR showed a loss or an irregularity of the inner and outer segment (IS/OS) line in the areas where the multifocal ERGs responses were reduced, and where the visual field defects were present. ${ }^{2,3}$ We have studied two patients with AZOOR at the convalescent stage by conventional ophthalmological examination and by SD-OCT, and present the morphology and thickness of the retinas at the convalescent stage.

\section{Case reports}

\section{Case I}

A 23-year-old myopic woman noticed photopsias and a dark spot in the inferior visual field of her right eye in May 2007. She was referred to another university hospital 10 days later, and her ocular and medical histories were normal at that time. Her best-corrected visual acuity (BCVA) was 0.9 OD and 1.5 OS, and the anterior segment was normal in both eyes. Ophthalmoscopy of her right eye showed a nearly 
normal fundus except for a white oval lesion of about one disc diameter near the temporal vascular arcade (Figure 1A). Perimetry detected an enlargement of the blind spot and deep scotomas in the inferior and nasal visual field of her right eye (Figure 1B). Fluorescein angiography showed that the white lesion was hypofluorescent. Magnetic resonance imaging (MRI) showed no abnormalities in the eyes and orbits, optic nerve, and brain. Her doctors decided to follow her without treatment.

After one year, she was examined at our hospital, the Matsumoto Dental University Hospital, and her main complaint was the dark spot in the inferior visual field. Her BCVA was 1.5 in both eyes, and the refractive errors were -5.75 diopters (D) OD and $-5.50 \mathrm{D}$ OS. The fundi were normal except for a small pigmented lesion in the right eye (Figure 2A). However, the scotoma that was connected to the blind spot and the nasal scotomas were still present (Figure 2B). The 30-Hz flicker ERGs, but not the full field ERGs were slightly reduced in the right eye (Figures $2 \mathrm{C}$ and 2D).

Examination by spectral domain optical coherence tomography (SD-OCT; Spectralis OCT; Heidelberg Engineering, Heidelberg, Germany) showed that the IS/OS line was not distinct with an absence of the middle line between the IS/OS and retinal pigment epithelium (RPE) in the temporal area of the macula but not in the fovea of the right eye (Figure 3A). Because it was not possible to conclude that the retina was thinner with only one scan, the retinal thickness was measured by using the volume scan of the SD-OCT. The results of this volume scan clearly showed a decrease in the mean retinal thickness in the superior and temporal areas of the nine areas corresponding to the Early Treatment Diabetic Retinopathy Study (ETDRS) areas (Figure 3B). ${ }^{4}$

\section{Case 2}

A 29-year-old woman was referred complaining of a sudden blurring of vision with photopsia of two days duration in her left eye. Her BCVA was 1.5 OD and 0.2 OS, and her refractive errors were -9.0 Ds OU. The anterior segment and ophthalmoscopic findings appeared normal (Figure 4A) except for an old focal retinal detachment without macular involvement in the inferotemporal area of the left eye. A strongly pigmented demarcation line was seen peripheral to the vascular arcade but probably was not associated with the acute central visual field loss. A dense visual field defect was detected in the superior field (Figure 4B). Fluorescein angiography showed no leakage or delayed filling in the macular lesion. The multifocal ERGs recorded from the macular area of the left eye were reduced (Figure 4C). Time-domain OCT (OCT3000; Zeiss, Oberkochen, Germany) showed an irregularity or loss of the IS/OS line in the fovea and a reduction in the thickness of the outer nuclear layer (ONL) (Figure 4D). The brain MRI findings were normal. Although oral prednisone (30 mg/day) was administered, the BCVA and the VF defect were unchanged.

Four years later, fundus examination showed a good macular reflex but the color of the deep retina or RPE in the macular area was changed. The localized retinal detachment was not altered (Figure 5). Goldmann visual field test showed an increase in the central scotoma but no
A

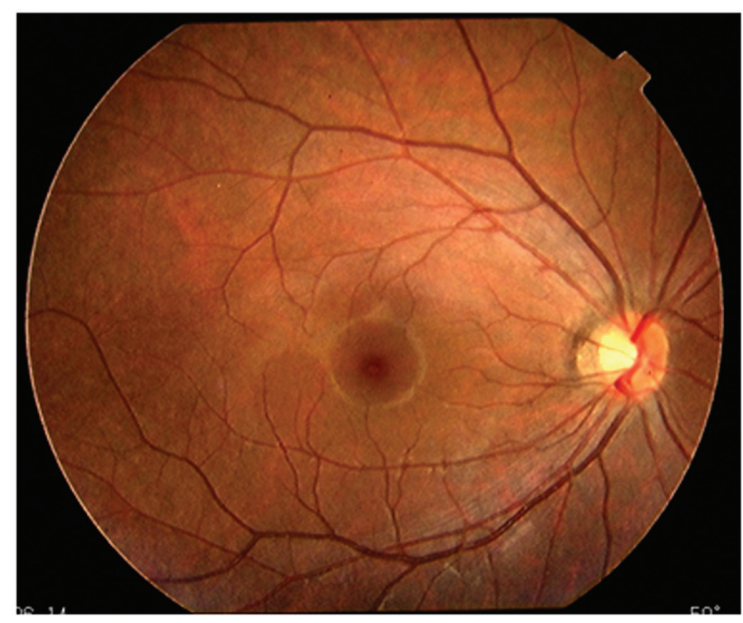

B

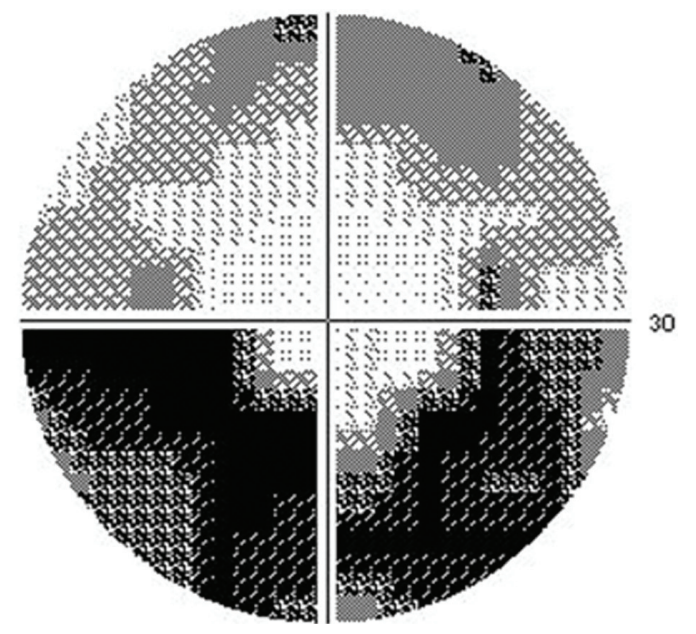

Figure I Case I Findings in a 23-year-old woman with AZOOR at onset (kindly provided by Professor Hiroyuki lijima of Yamanashi University Hospital). A) Fundus photograph of posterior pole of the right eye. Fundus appears normal. B) Visual field defect characterized by enlargement of blind spot and deep scotomas in the inferior and nasal visual field of her right eye. 
significant change of the superior defect (data not shown). Multifocal ERGs were not available in our hospital, but the full-field and 30-Hz flicker ERGs were reduced (data not shown).

SD-OCT showed a greater loss of the IS/OS line, and an alteration of the outer nuclear layers especially in the inferior macular area (Figure 6A). The retinal thickness was markedly reduced with a decrease of both the outer and inner nuclear layers (Figure 6B).

\section{Discussion}

Both of our myopic female patients with AZOOR presented with reduced vision and visual field defects. They both reported photopsia, and their fundus was essentially normal. The ERG changes were present only in the affected eye. The diagnosis of AZOOR was based on these characteristic features of AZOOR as described by Gass. ${ }^{5}$
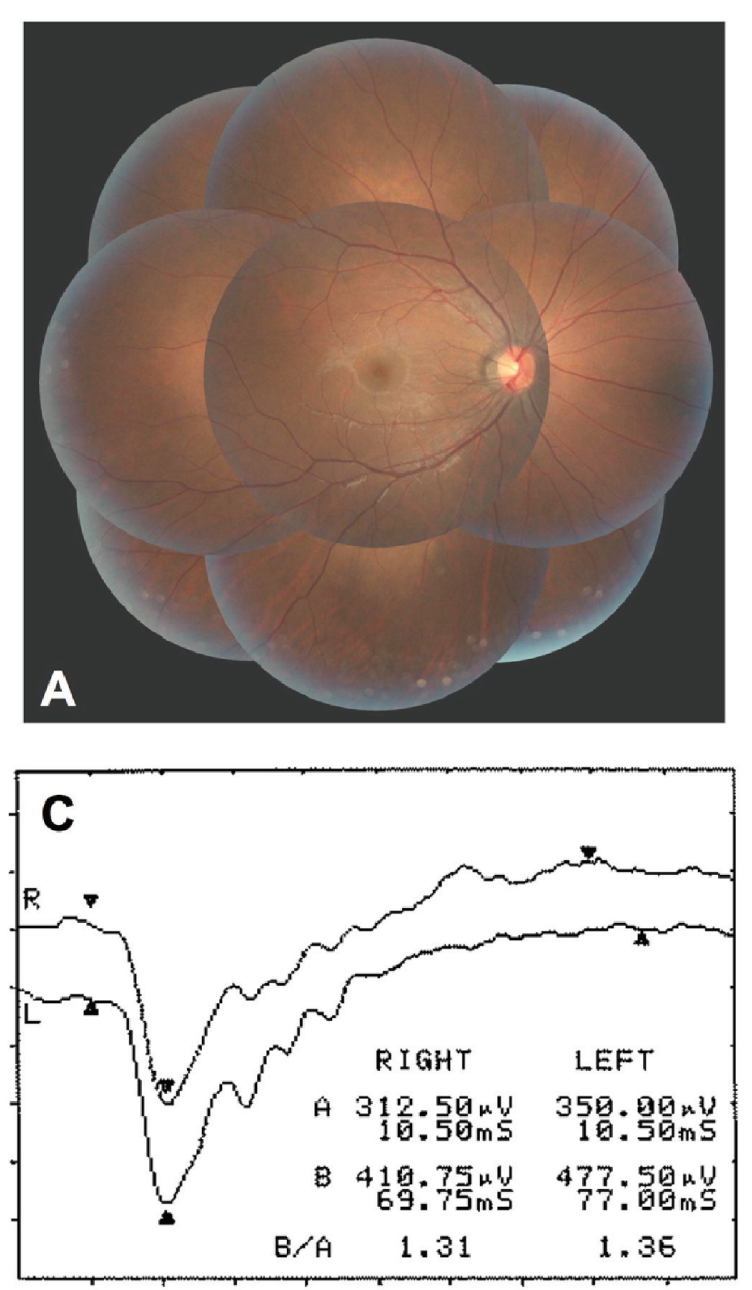

OCT revealed a loss or irregularity of the IS-OS line in the area where outer retinal lesions were detected, ${ }^{2,3}$ and the regions where the IS-OS line was abnormal corresponded with the areas where the visual field defects were present and the mfERGs were reduced. ${ }^{2}$ The OCT findings supported the hypothesis by Gass that the primary lesion of AZOOR is a degeneration of the photoreceptor outer segments.

An earlier time-domain OCT study found a reduction in the thickness of the ONL in the macular area in only one of five cases of AZOOR. ${ }^{2}$ However, an absence or irregularity of the IS-OS line was observed in all cases. ${ }^{2}$ Recent higher resolution images obtained by the SD-OCT ${ }^{3,6}$ also showed a thinning of the affected retina, but the images were obtained by a single scan. A better picture of the retinal thickness of the macula with high reproducibility can be obtained by using the volume scan of the Spectralis SD-OCT. ${ }^{7}$ With this instrument, the mean retinal thickness map is determined from
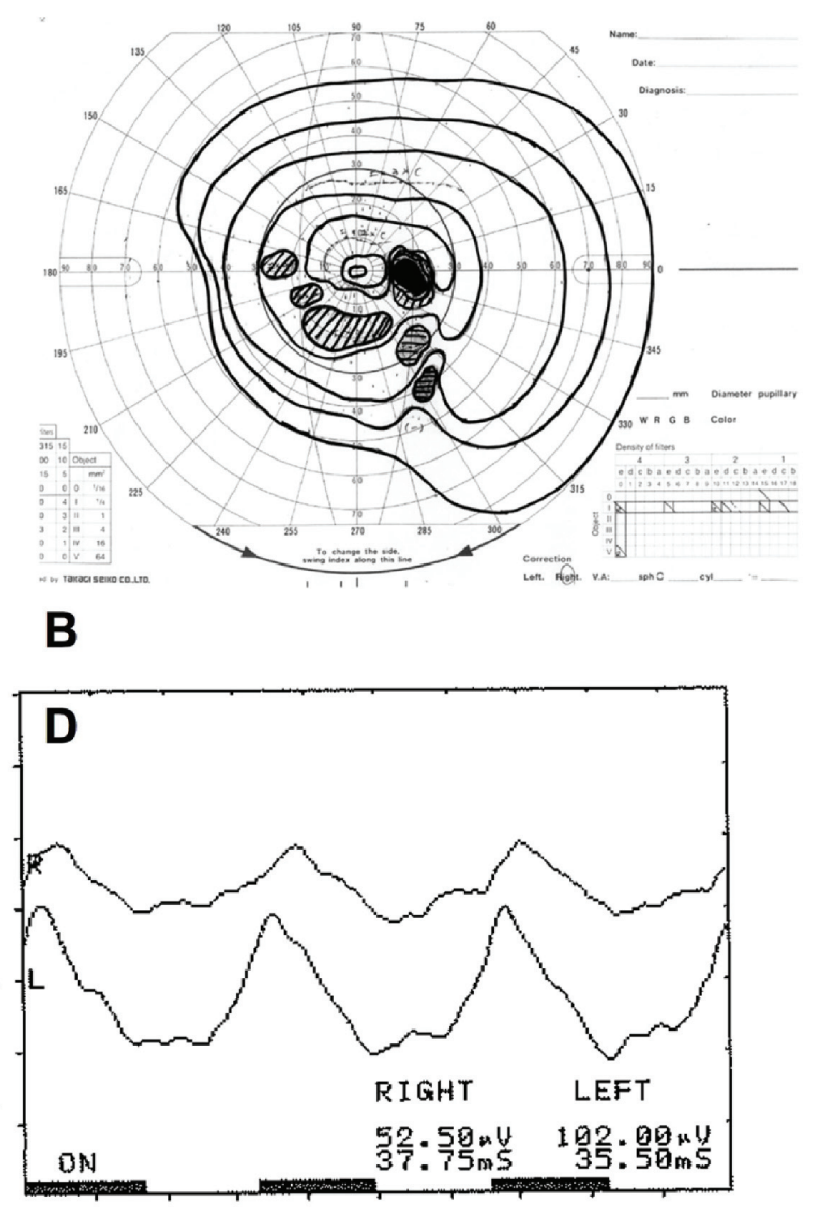

Figure 2 Case I Findings one year later. A) Nearly normal panoramic fundus photograph of the right eye. B) Persistent visual field defect characterized by enlargement of blind spot and scattered scotomas. C) Single-flash full-field electroretinograms (ERGs) were within the normal limits in both eyes. D) Flicker (30-Hz) ERG from both eyes showing a reduction in the amplitude and significant phase delay in the amplitude in the right eye. 


\section{A}

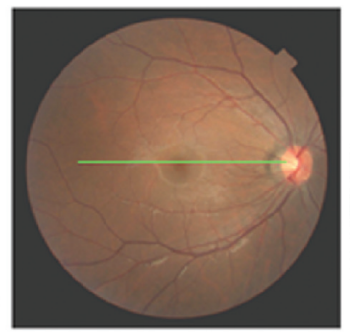

$\mathbf{T}$

$\mathbf{N}$
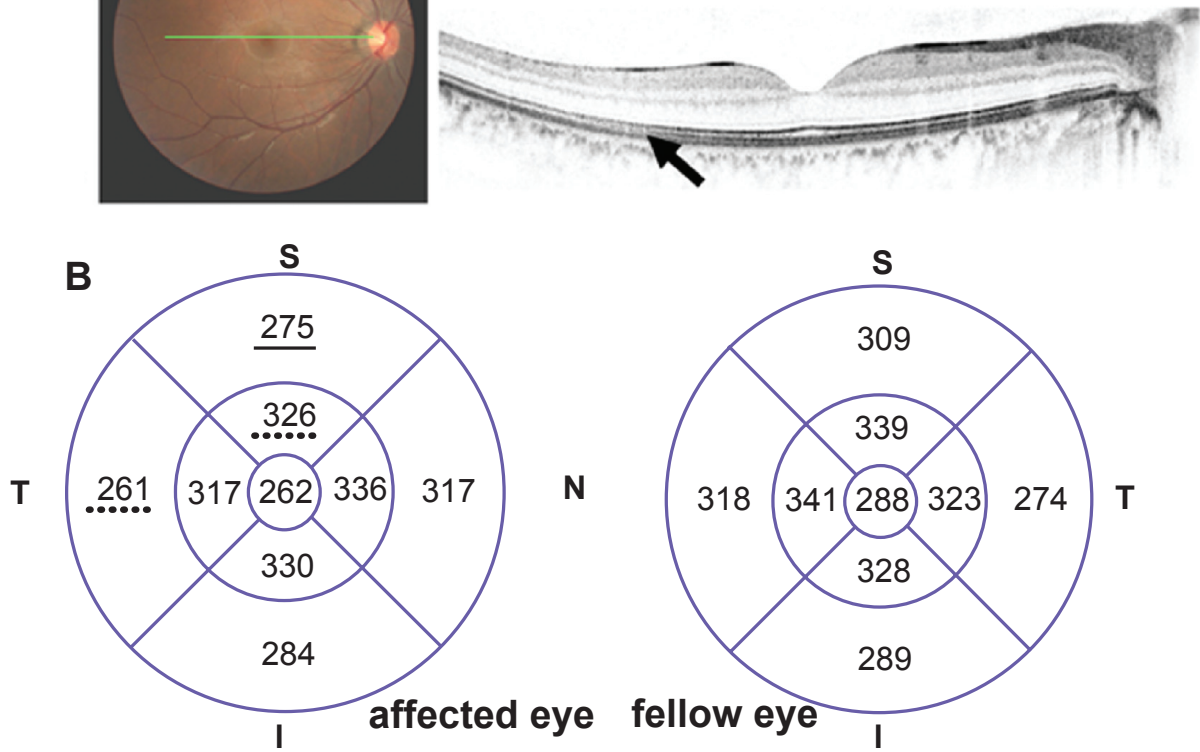

Figure 3 Case I Findings one year later. A) Optical coherence tomographic image of a 6-mm horizontal scan showing the loss of middle line and slightly decreased IS/OS line (arrow). B) Retinal volume map of the right and left eyes. The retina in right eye is thinner than that of the fellow eye by volume scan analysis. Values underlined = reduced by $<10 \%$ and by dotted lines by $<3 \%$.

Abbreviations: S, superior;T, temporal; I, inferior; N, nasal.

A

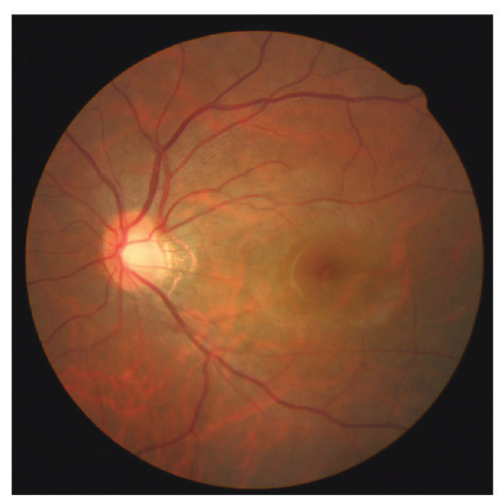

fellow eye
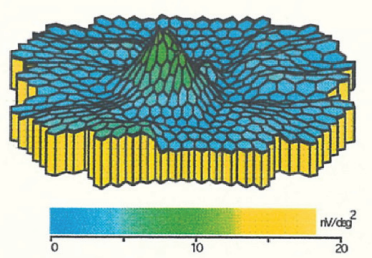

C

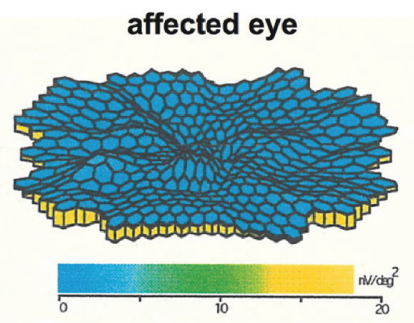

Figure 4 Case 2 Findings in a 29-year-old woman with AZOOR at onset. A) Nearly normal fundus photograph of posterior pole of the left eye. B) Dense superior visual field defects. C) Topographic map of multifocal ERGs showing markedly reduced responses in the macular area. D) Time-domain optical coherence tomography image showing a reduction of the outer nuclear layer.

Abbreviations: S, superior; I, inferior.

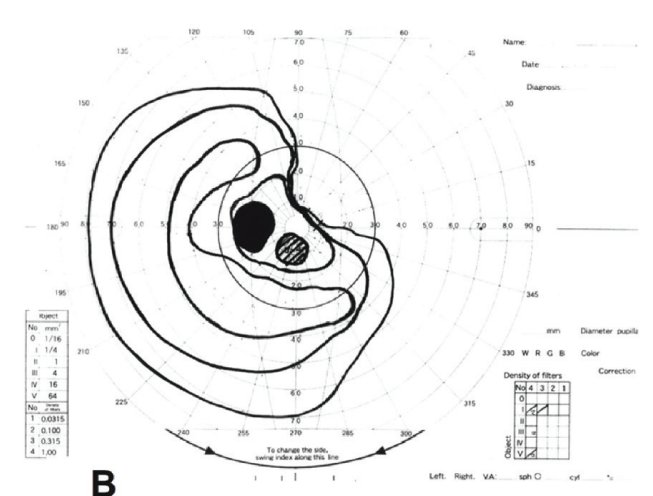

B

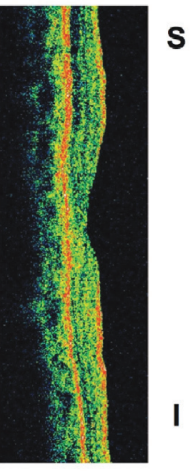




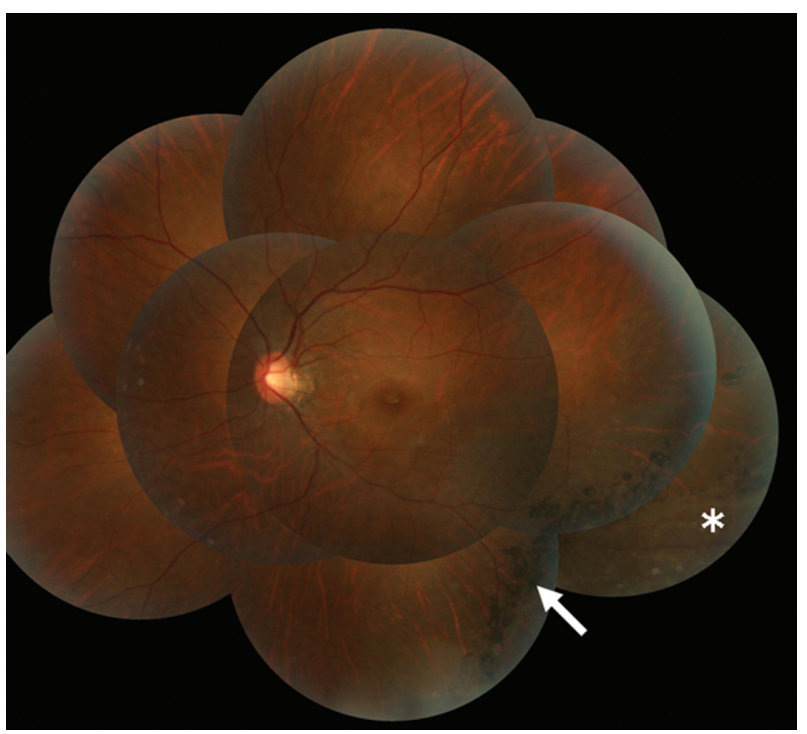

Figure 5 Case 2 Panoramic fundus photograph of left eye four years after onset. The localized retinal detachment $(*)$ is well-demarcated by pigmentation (arrow). nine sections in three concentric circles of 1,3 , and $6 \mathrm{~mm}$ diameters around the foveola. The procedures we used were similar to that reported by the ETDRS. ${ }^{4,7}$

In our two cases, the retina in the involved area was thinner than that in the healthy areas (Figures 2 and 4). The reduction in the thickness of the retina in Case 1 was mild, viz., a 3\%-20\% reduction of the thickness of a corresponding area of the fellow eye. The reduction was more severe in Case 2, viz., $>40 \%$ reduction. Similar findings of a decrease of the ONL and thinning of INL has been found by SD-OCT in at least two of three cases. ${ }^{3}$ This decrease in the thickness of the ONL plus thinning of INL in severe cases of AZOOR may reflect secondary degeneration after damage of the outer segments.

Our findings support an earlier suggestion that the photoreceptor outer segments were the primary sites of the disease process in AZOOR. ${ }^{2}$ Our findings indicated that damage of
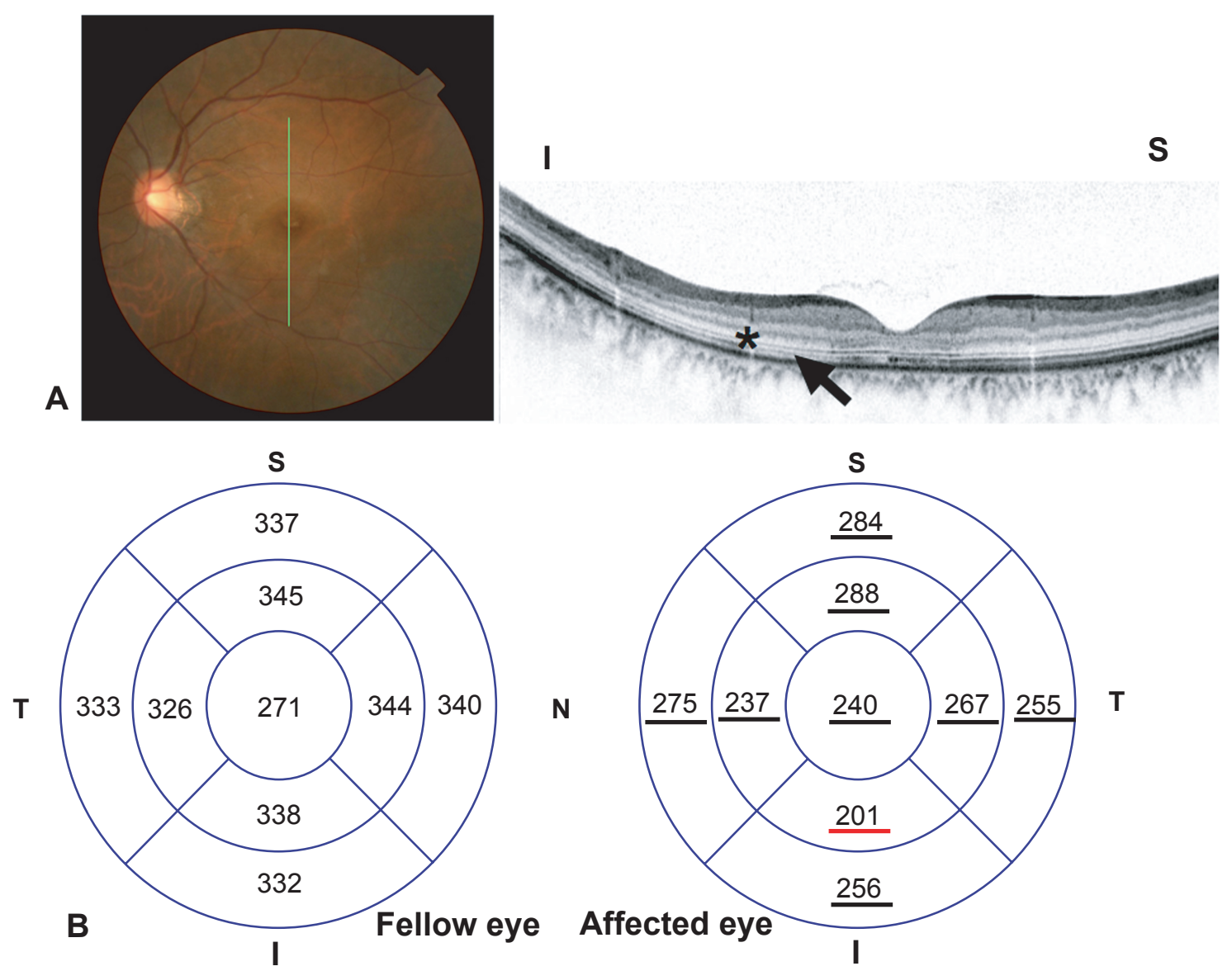

Figure 6 Case 2 Findings four years after onset. A) Spectral domain optical coherence tomography image of a 6-mm vertical scan showing the attenuated IS/OS line (arrow) and severely decreased outer nuclear layer $(*)$, outer plexiform layer and inner nuclear layer. B) Decreased retinal thickness in left eye compared with fellow eye by volume scan analysis. Underlines; decreased less than $10 \%$ or red line; less than $40 \%$.

Abbreviations: S, superior; T, temporal; I, inferior; N, nasal. 
the photoreceptors can cause secondary degeneration of not only the ONL but also the INL.

\section{Disclosure}

No author has any proprietary or conflict of interest in this work. KO had full access to all data in the study and responsibility for integrity of the data and accuracy of the data analysis. We thank Prof. Hiroyuki Iijima of Yamanashi University Hospital for providing the data of Case 1 at the onset. We thank Prof. Duco Hamasaki of the Bascom Palmer Eye Institute for editing the manuscript.

\section{References}

1. Gass JD, Agarwal A, Scott IU. Acute zonal occult outer retinopathy: a long-term follow-up study. Am J Ophthalmol. 2002;134:329-339.

2. Li D, Kishi S. Loss of photoreceptor outer segment in acute zonal occult outer etinopathy. Arch Ophthalmol. 2007;125:1194-1200.
3. Spaide RF, Koizumi H, Freund KB. Photoreceptor outer segment abnormalities as cause of blind spot enlargement in acute zonal occult outer retinopathy-complex diseases. Am J Ophthalmol. 2008;146:111-120.

4. Photocoagulation for diabetic macular edema. Early Treatment Diabetic Retinopathy Study report number 1. Early Treatment Diabetic Retinopathy Study research group. Arch Ophthalmol. 1985;103:1796-1806.

5. Gass JD. Acute zonal occult outer retinopathy. Donders lecture: The Netherlands Ophthalmology Society, Maastricht, Holland, June 19, 1992. J Clin Neuro Ophthalmol. 1993;13:79-97.

6. Zibrandtsen N, Munch IC, Klemp K, Jorgensen TM, Sander B, Larsen M. Photoreceptor atrophy in acute zonal occult outer retinopathy. Acta Ophthalmol. 2008;86:913-916.

7. Menke MN, Dabov S, Knecht P, Sturm V. Reproducibility of retinal thickness measurement in healthy subjects using spectralis optical coherence tomography. Am J Ophthalmol. 2009;147:467-472.
Clinical Ophthalmology

\section{Publish your work in this journal}

Clinical Ophthalmology is an international, peer-reviewed journal covering all subspecialties within ophthalmology. Key topics include: Optometry; Visual science; Pharmacology and drug therapy in eye diseases; Basic Sciences; Primary and Secondary eye care; Patient Safety and Quality of Care Improvements. This journal is indexed on

Submit your manuscript here: http://www.dovepress.com/clinical-ophthalmology-journal

\section{Dovepress}

PubMed Central and CAS, and is the official journal of The Society of Clinical Ophthalmology (SCO). The manuscript management system is completely online and includes a very quick and fair peer-review system, which is all easy to use. Visit http://www.dovepress.com/ testimonials.php to read real quotes from published authors. 